\title{
Contrôle de validité de la prédétermination des pertes de charge d'une galerie par circulation d'air
}

\section{Checks on the validity of the method of predetermining head losses by circulating air}

\author{
PAR P. BOURGUTGNON
}

INGENIEUR DES ARTS ET MANUFACTURES,

CHEF DE LA DIVISION « ESSAIS EXTERIEURS \$ DU SERVICF DYS

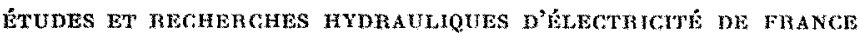

\begin{abstract}
Quatre galeries ont fait l'objet de mesures successives de pertes de charge par circulation d'air et d'eau. Pour deux d'entre elles (Vénéon et Le Ponget), les conditions de similitude theorique étant parfaitement respectées (écoulement en charge avec nombre de Regnolds supériear da la valeur critique), les pertes de charge $s^{2} y$ sont révélées égales $\dot{a} \pm \mathbf{5} \%$ près ponr les denx éconlements.

Les essais à l'air effectués dar's la galerie de Nentilla correspondent da un nombre de Reynolds très peu inférieur à la valeur critique: toutefois, les résultats des deux essais sont encore très concordants. Pour la galerie de Peyrat-le-Château, la circulation d'eau s'effectue à écoulement libre: les résnltats obtenus pour les essais à l'air on't néanmoins permis de prédéterminer, $\dot{a}$ moins de $10 \mathrm{~cm}$ près, la ligne d'eau effectivement réalisée.
\end{abstract}

Au cours du $3^{\circ}$ Congrès de l'Association Internationale de Recherches Hydrauliques, M. REmENIERAs présentait une méthode de * prédétermination de la perte de charge dans une canalisation d'eau sous pression à partir de celle mesurée sur la même canalisation parcourue par de l'air ». Cette communication était accompagnée des résultats d'essais de contrôle de la validité de la méthode effectués dans la galerie d'amenée R.G. de l'usine hydroélectrique de PontEscoffier.

Depuis cette époque, sept essais ont été effectués sur la demande de Régions d'Equipement de l'Electricité de France en vue d'une meilleure discrimination dans le choix de la répartition

\begin{abstract}
Head loss measurements were made on four tunnels through which water and air were circulated successively. On two of them (Vénéon ard Le Pouget) exact theoretical similitude conditions were observed (flow under pressure with a Relnolds number greater than the critical value) and identical head losses to within about $\pm 5 \%$ were found for both flows.

The air tests carried out on the Nentilla tunnel were at a Reynolds number very slightly lower than the critical wahe; nevertheless there mas still a very considerable degree of agreement between the results of both tests water was circulated in the Peyrat-le-Chateau tunnel under free flow conditions, the results given by the air tests made it possible nevertheless forecast the eventual surface profile to within about $10 \mathrm{cms}$.
\end{abstract}

des revêtements d'une galerie en cours d'exécution. Trois d'entre elles seulement ont pu faire l'objet d'essais avec circulation d'eau dans des conditions à peu près comparables à celles réalisées au cours des essais de circulation d'air. Cela tient à ce que les tronçons de mesure ont été partiellement ou totalement revêtus à la suite de nos essais, soit parce que les pertes de charge escomptées paraissaient trop élevées, soit par suite de la tenue insuffisante du terrain en cerw taines sections.

L'objet de la présente communication est de présenter les résultats obtenus au cours de ces essais de contrôle de la méthode. 


\section{I. - GALERIE D'AMENEE R. G. DE L'USINE HYDROELECTRIQUE DE PONT-ESCOFFIER}

\section{(Aménagement du Vénéon)}

Les résultats de ces essais ont été exposés en annexe de la communication de M. Remenieras. Les figures 1,2 et 3 reproduisent les dispositions de mesures utilisées pour ces essais : les débits étaient mesurés au moyen d'une batterie de tubes de Pitot montés sur un bras diamétral tournant installé dans la section circulaire d'une buse de $800 \mathrm{~mm}$ de diamètre aménagée dans une conduite en béton reliant la prise d'eau au dessableur installé à l'entrée de la galerie.

Trois tronçons de pertes de charge avaient été aménagés, dont un seul pouvait être utilisé pour les mesures par circulation d'air et d'eau. Le tronçon $\mathrm{A}$, de $174 \mathrm{~m}$ de longueur, ne pouvait être complètement mis en charge pour l'écoulement d'eau. Quant au tronçon $\mathrm{C}$, de $673 \mathrm{~m}$ de longueur, il était partiellement recouvert par les eaux s'infiltrant par les fissures de la galerie, dont l'épuisement ne pouvait être envisagé (les rentrées d'eau les plus importantes étaient localisées à plus de $1 \mathrm{~km}$ de l'entrée de la galerie). De ce fait, les conditions d'écoulement de l'air et de l'eau ne pouvaient pas être comparables, la présence d'eau sur le radier modifiant la section utile offerte au passage de l'air ainsi que la rugosité du radier.

Seul, le tronçon $B$ offrait, sur une longueur de $620 \mathrm{~m}$, des conditions d'écoulement semblables pour les deux fluides. Encore fallait-il, pour obtenir ce résultat, épuiser les eaux d'infiltration, concentrées à l'amont de ce secteur, au moyen de batardeaux et de groupes motopompes, dont l'installation est représentée dans la figure 2.

Les prises de pression statiques installées sur des trépieds visibles sur la figure 2 étaient raccordées par des canalisations en tubes d'aluminium à 2 postes de mesure équipés de 2 manomètres différentiels à colonnes « air-eau » ou «air-alcool » à tubes inclinés, d'une sensibilité de lecture de $1 / 20 \mathrm{~mm}$. La disposition des canalisations de raccordement des prises de pression aux postes de mesure était réalisée de telle sorte que les pertes de charge d'un troncon ou la somme des pertes de charge dans 2 tronçons successifs pouvait être alternativement mesurée par l'un et l'autre des 2 postes. Pour les essais à l'air, les recoupements ainsi obtenus étaient excellents; les pertes de charge déduites des relevés aux 2 postes s'avéraient égales à moins de $5 \%$ près. Les pertes de charge mesurées au cours de la circulation d'eau étaient relativement moins cohérentes: l'impossibilité de pénétrer dans la galerie en charge pour manœuvrer les

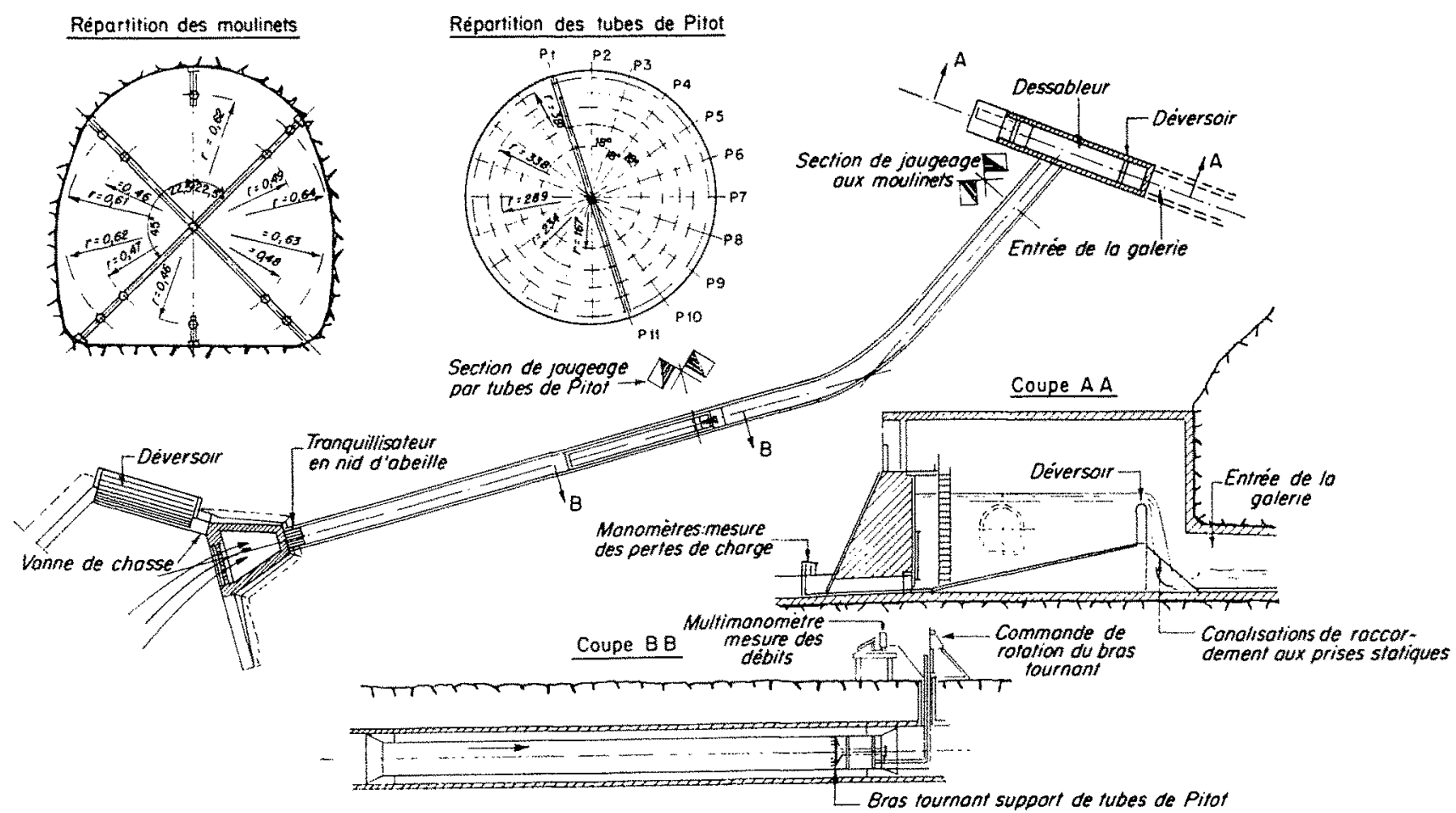

Fic. 1. - Installation pour mesure des dẻbits. 


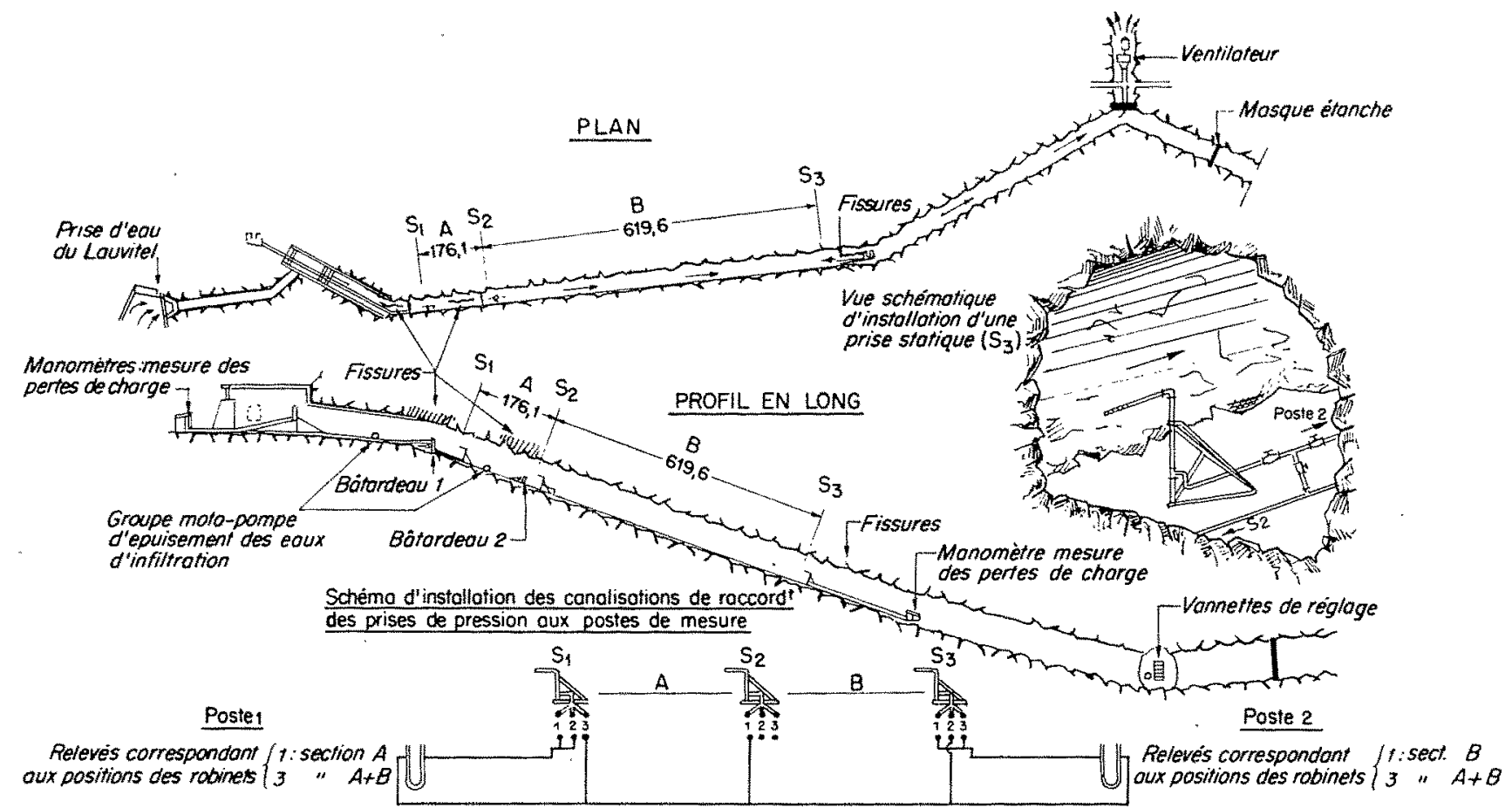

Fig. 2. - Installation de mesure des pertes de eharge dans l'air.

robinets de commutation des prises de pression aux manomètres, exigeait la vidange de la galerie entre 2 séries d'essais ce qui, compte tenu du temps de remplissage, demandait deux à trois jours pendant lesquels la purge d'air des canalisations de mesure devait être maintenue. Ce fait, joint à la grande longueur des canalisations (la canalisation commune aux 2 postes de mesure avait une longueur de $2 \mathrm{~km}$ environ) explique sans doute l'écart entre les 2 séries de mesure successives de la somme des pertes de charge dans les troncons $B$ et $C$. Cet écart atteint $2 \mathrm{~mm}$ d'eau pour le point à plus grand débit, soit $10 \%$ environ de la perte de charge dans le tronçon $B$.

Les résultats des mesures ainsi effectuées sur le tronçon $B$ sont rassemblés dans la figure 4 où pour chaque point de mesure les pertes de charge en $\mathrm{mm}$ d'eau ont été reportées en fonction de la hauteur dynamique en mm d'eau

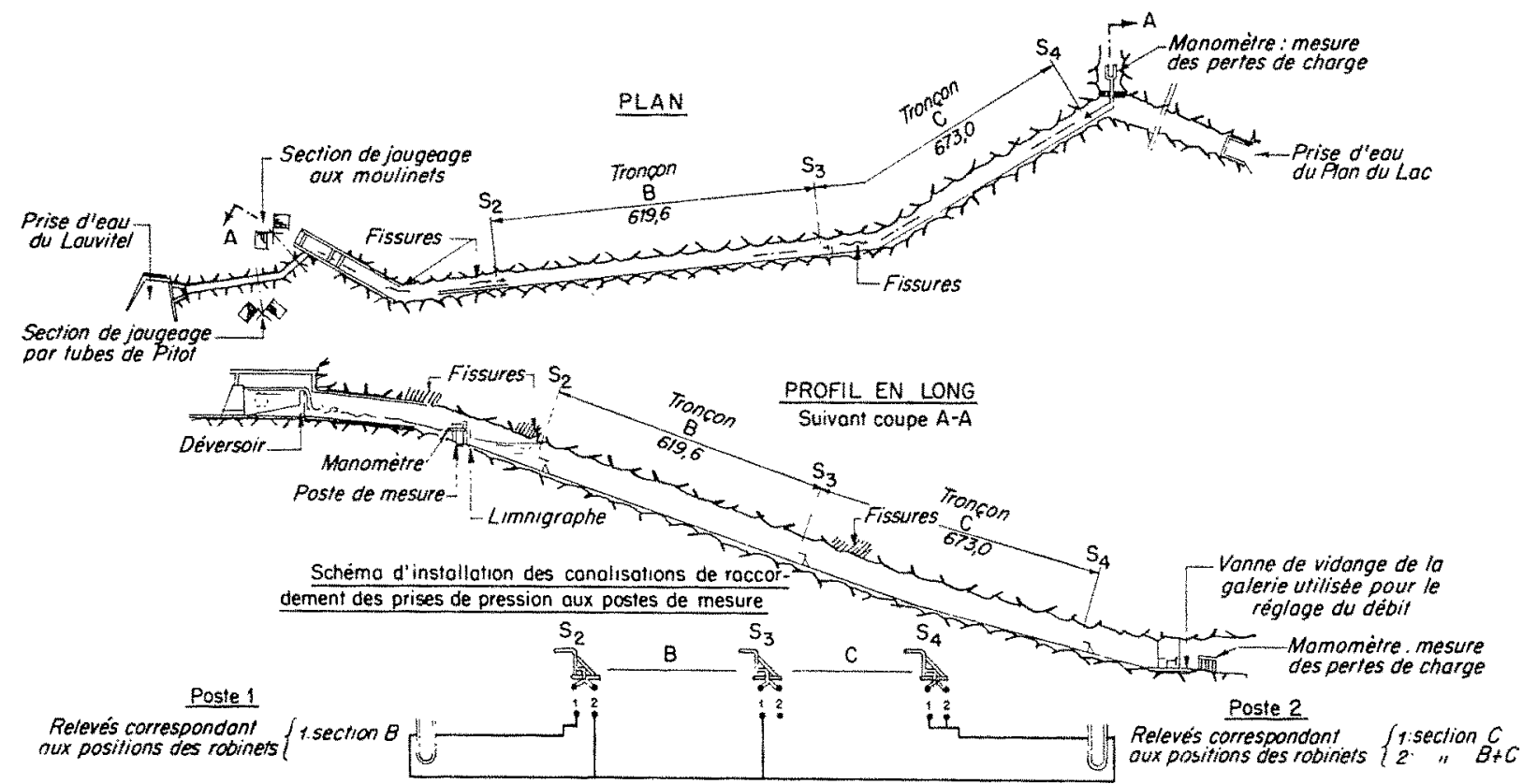

Fig. 3. - Installation de mesure des pertes de charge dans l'eau. 


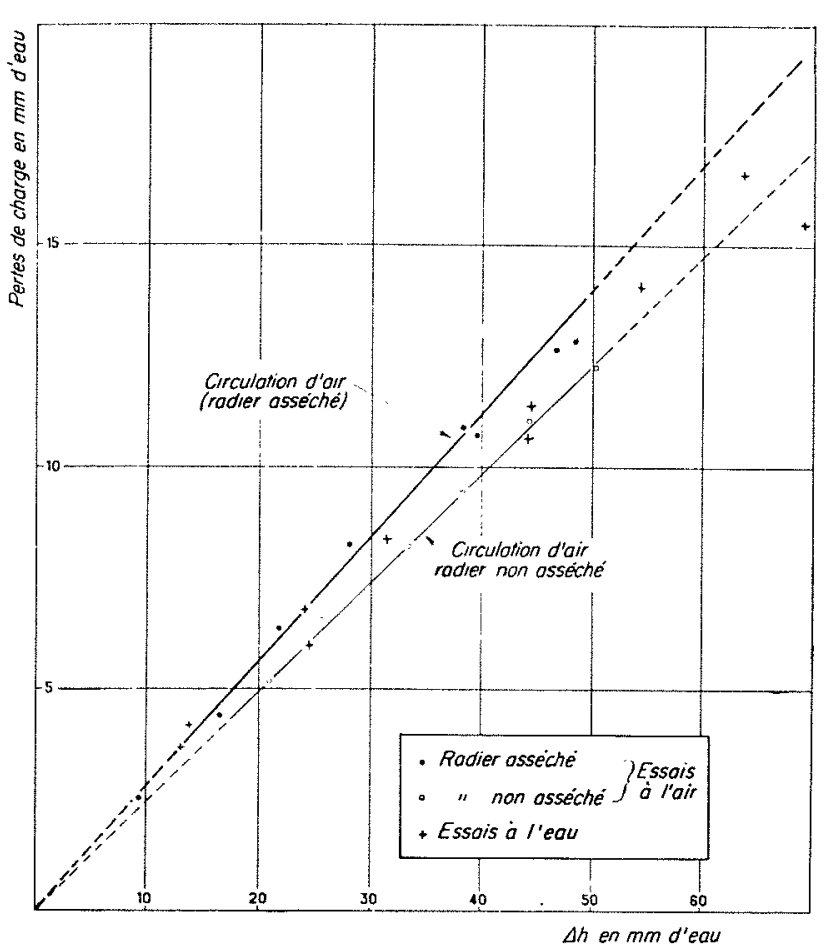

Fra. 4. - Galerie R. G. de l'usine de Pont-Escoffier.

correspondant à la vitesse moyenne dans la section de mesure des débits.

Par suite de la rugosité relativement importante de cette galerie, le régime turbulentrugueux était largement réalisé à tous les régimes pour les 2 types d'écoulement. De ce fait, le coefficient $\lambda$ de la formule universelle des pertes de charge devait être constant et l'on pouvait écrire aussi bien pour l'air que pour l'eau :

$$
\xi=\lambda \frac{\mathrm{V}_{0}}{2 g} \int_{0}^{x}\left(\frac{\lambda}{\mathrm{D}_{k}} \cdot \frac{\mathrm{S}_{0}}{\mathrm{~S}}\right)^{2} d l
$$

en désignant par $V_{0}$ et $S_{0}$ la vitesse moyenne et l'aire de la section de jaugeage, et par $S$ l'aire d'une section courante de la galerie à une distance $l$ de l'origine du troncon considéré $\left(\mathrm{D}_{h}\right.$ représente le diamètre de frottement, égal à 4 fois le rayon hydraulique).

Le terme sous le signe somme est un nombre sans dimension, ne dépendant que des caractérisliques géométriques de la galerie et de la section de jaugeage et dont la valeur est la même pour tous les écoulements. En le désignant par G, on peut écrire :

$$
\lambda=\frac{\xi}{\frac{\mathrm{V}_{0}{ }^{2}}{2 g}} \times \frac{1}{\mathrm{G}}
$$

ou, en désignant par $\delta h_{0}$ la hauteur

$$
\begin{gathered}
\frac{\mathrm{V}_{0}{ }^{2}}{2 g} \\
\frac{\zeta}{\delta h_{0}}=\lambda \mathrm{G}
\end{gathered}
$$

Dans cette relation, le poids spécifique du fluide n'intervient pas $\left(^{*}\right)$ à la condition que les pertes de charge et les dénivellations $\delta h_{0}$ soient exprimées au moyen d'une même unité.

Sur le graphique de la figure 4 , on peut constater que les points d'essais avec circulation d'air se répartissent avec une faible dispersion (de l'ordre de 0,5 à $1 \mathrm{~mm}$ d'eau) autour de 2 droites distinctes. Cela provient de la grande rugosité du radier de la galerie étudiée; sans précaution spéciale celui-ci est recouvert d'une nappe d'eau qui constitue pour l'air une paroi relativement unie. En rejetant à l'extérieur les débits de rentrée d'eau qui se produisent à l'entrée du troncon A, la suppression de cette paroi d'eau en écoulement d'air rendait au radier sa rugosité naturelle, ce qui contribuait à augmenter la perte de charge, malgré la légère majoration de section $\left(^{\star *}\right)$.

Peut-être faut-il chercher une explication semblable à la dispersion des points de mesure avec circulation d'eau. La galerie n'étant qu'à très faible charge et la mise en eau ne s'effectuant que peu de temps avant les essais, il persistait une quantité d'air non négligeable dans les anfractuosités existant dans le ciel de la galerie. Dis lors, le coefficient de rugosilé étail en dépendance de la surface d'air emprisonnée dans ces alvéoles, c'est-à-dire, en définitive de la pression d'eau en chaque section. Compte tenu de la surface importante du plan d'eau précédant le troncon mis en charge, il n'aurait guère été possible de régler cette charge à une valeur constante. De ce fait, comme la rugosité naturelle du ciel de la galerie est beaucoup plus importante que celle du radier, plus ou moins nivelé par les matériaux fins transportés, une différence de charge de 1 mètre d'eau pouvail entrainer pour l'eau des

(*) En fait, cette relation suppose que le poids spécifique du fluide soil le mème dans la section de jaugeage et dans le tronçon de galerie où les pertes de charge sont relevées. Cette condition ne pouvait être entisument réalisée pour les essais à l'air, en raison de la valiation du degré hygrométrique et de la température de l'air entre l'entrée de la galerie et les tronçons de mesure. II en résultait une légère correction qui, dans le cas le plus défavorable, ne dépassait pas $6 \%$.

(*) Compte tenu des relevés de protil effectués et en admettant pour le frottement « air-eau $»$ un coefficient de rugosité de 70 , nous avons retrouvé sensiblement l'écart entre les pertes de charge observes dans ces deux séries d'essais par la formule d'Einstein applicable aux galeries à parois mixtes. 


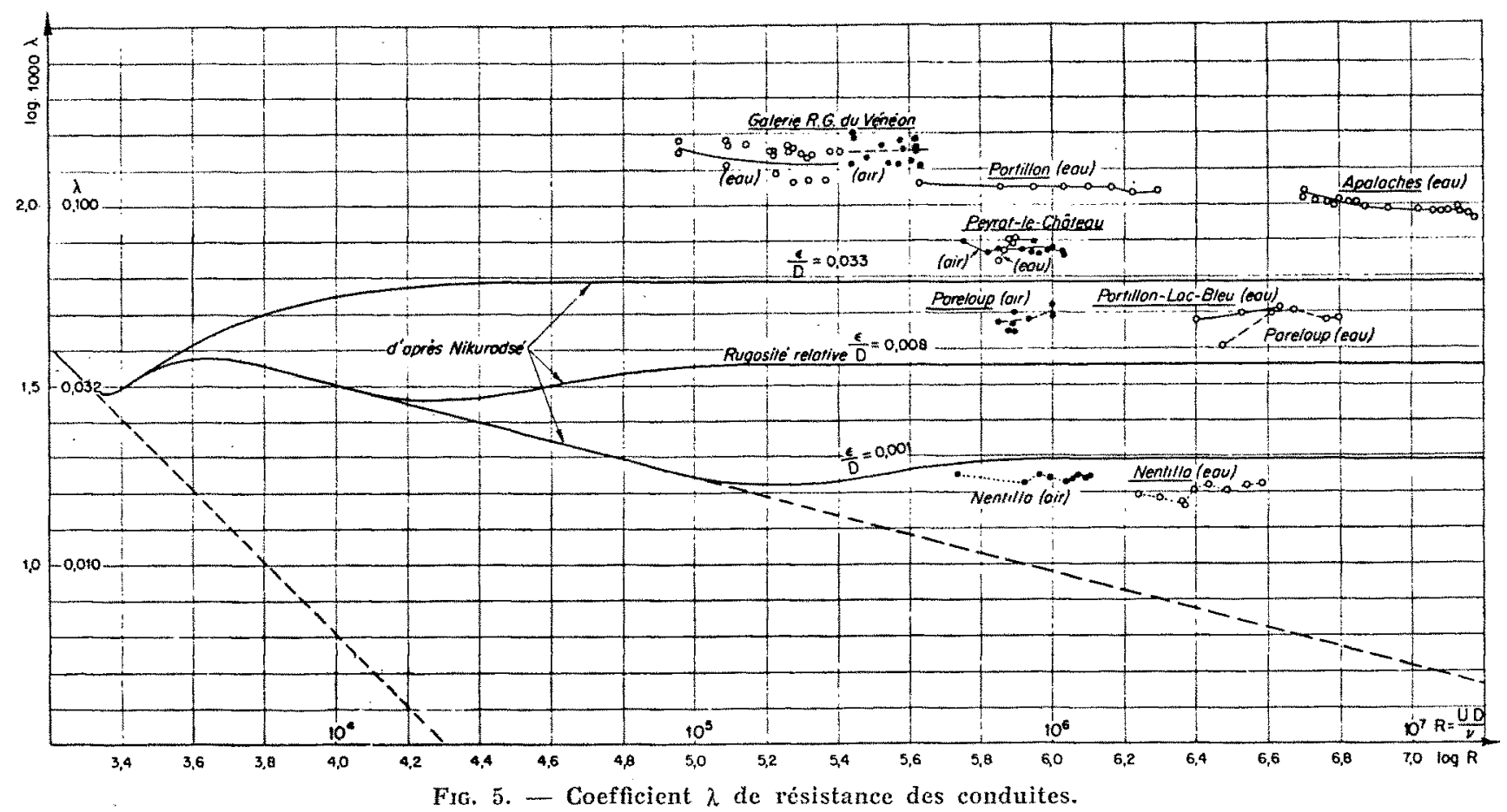

variations de rugosité d'ensemble comparables à l'asséchement du radier pour les essais à l'air.

Tels quels, les points expérimentaux de circulation d'eau se placent avec une dispersion moyenne de l'ordre de $\pm 5 \%$ autour de la droite correspondant à la perte de charge dans l'air, radier non asséché.

Les coefficients $\lambda$ de la formule universelle des pertes de charge relatifs à ces différents essais ressortent aux valeurs suivantes, reportées dans la figure 5 :

Rugosité en écoulement d'air :

Radier asséché (brut) ...... 0, 0,144

Radier non asséché (lisse).. 0,128

Rugosité en écoulement d'eau. . 0,126
Le coefficient $\lambda$ moyen avec circulation d'eau est donc en moyenne de $2 \%$ environ inférieur à celui obtenu avec l'air lorsque le radier est « lissé » par les eaux d'infiltration.

Ces valeurs sont d'autant plus comparables qu'un relevé de profils très serré sur une dizaine de mètres nous a permis de constater que la surface d'air emprisonnée dans le ciel de la galerie était du même ordre de grandeur que la surface du radier recouverte par les eaux d'infiltration.

Les deux méthodes de mesure conduisent donc à des valeurs très voisines, l'écart entre les résultats s'avèrent inférieurs à l'erreur probable de mesures que nous avons évalucé à $\pm 5 \%$ environ.

\section{II. - GALERIE DE “ PARELOUP - MAS-ROUS " (Equipement du Pouget)}

Nous nous étendrons moins longuement sur les galeries pour lesquelles les essais avec circulation d'air ont déjà fait l'objet d'une communication à la Société Hydrotechnique de France.

Les mesures comparatives de pertes de charge avec circulation d'air et d'eau ont été effectuées dans le tronçon A B (fig. 6) de la galerie Pare-
loup-Mas-Rous de $170 \mathrm{~m}$ de longueur, dans leque] les pertes de charge avaient été mesurées à deux reprises par circulation d'air avant et après le revêtement du radier et des piédroits. Ainsi que cela avait été exposé dans la communication précitée $\left.{ }^{\star}\right)$, ce revêtement partiel n'avait apporté

(*) Génie Civil dn 15 mars 1953. 


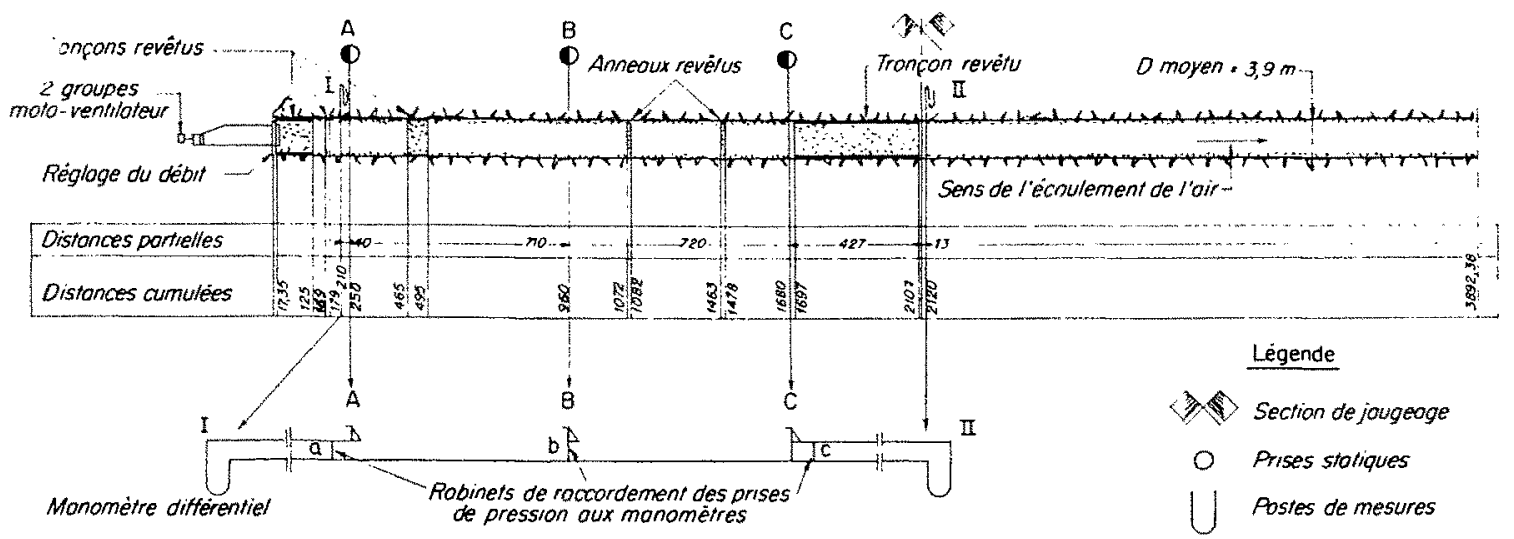

Schémo des connections entre "prses" et "monomètres"

Fid. 6. - Galerie Pareloup-Mas-Rous.

Schéma général d'installation des postes de mesures.

aucune réduction de pertes de charge, la diminution de rugosité ne faisant que compenser la réduction de section mouillée.

C'est dans ce tronçon partiellement brut qu'onl été réalisés les essais par circulation d'eau, les débits étant mesurés à partir des indications de moulinets dans la conduite forcée de l'usine d'Alrance et de l'ouverture des distributeurs de la turbine de cette usine. Les pertes de charge étaient relevées au moyen de manomètres différentiels

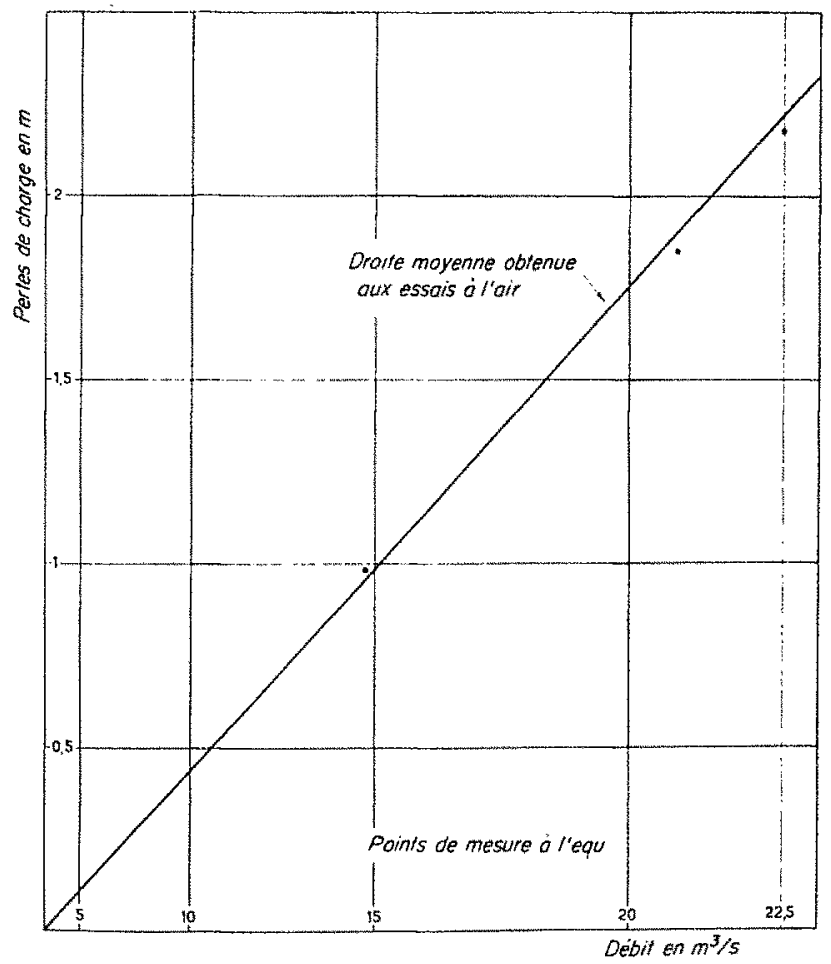

Fig. 7. - Galerie de Pareloup-Mas-Rous. à colonnes «aì-eau ». Les résultats de ces relevés sont représentés dans la figure 7 dans laquelle les pertes de charge en mètres d'eau sont reproduites en fonction du carré des débits : à titre de comparaison nous avons fait figurer sur ce graphique la droite moyenne des pertes de charge résultant des essais à l'air $\left({ }^{*}\right)$. On peut constater ainsi que les points de mesure des essais avec circulation d'eau sont peu éloignés de cette droite.

La concordance entre los pertes de charge mesurées avec les deux fluides est encore mieux mise en évidence dans la figure 5 précitéc. Les coefficients $\left({ }^{*}\right) \lambda$ de pertes de charge résultant de ces essais ressortent en effet à : et à :

- 0,0481 pour les essais par circulation d'air,

\section{- 0,0489 pour les essais par circulation d'eau.}

L'écart entre les coefficients de rugosilé déduits des 2 séries d'essais est done de 1,7\%, inférieur à l'approximation de chacune des mesures, évaluée au micux à $\pm 5 \%$.

(**) Gette droite diffère légèrement de celle dédnite des essais relatés dans larticle précite. Nous nous sommes en effet apercus entre temps d'une creut tlissé dans nos relevés de débits par circulation d'air due a une incertitude dans le tarage des moulinets hydrométriques en soufflerie. Cette erreur n'a pu être décelée qu'au cours des essais ultérieurs effectués par circulation d'air dans les galeries, grâce à l'emploi d'un anémomètre concurremment avec les moulinets.

$\left.{ }^{\star}\right)$ Les dimensions géométriques intervenant dans la détermination de ce cocficient sont évidemment les mêmes pour les deux fluides; l'erreur sur leur valeur v'entre done pas dans la comparaison des résnltats cbtenus au cours des deux séries de mesure. 


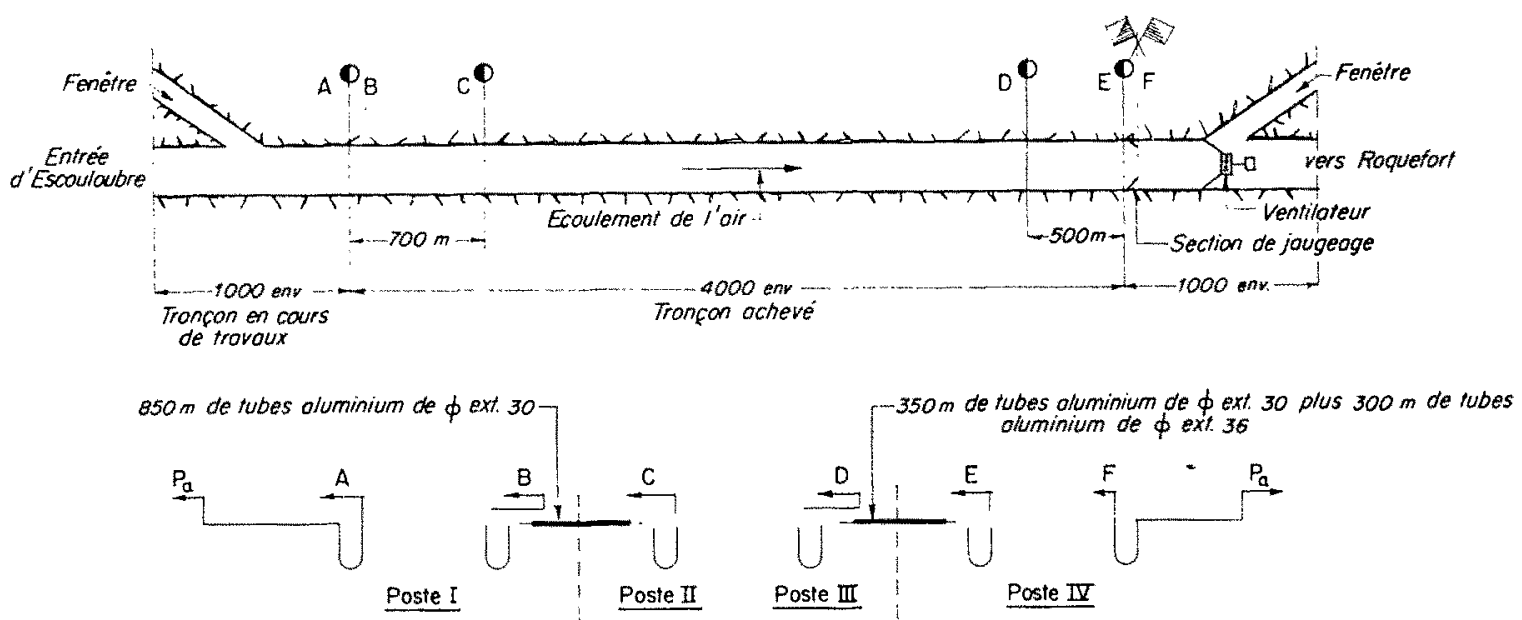

Fig. 8. - Gillerie de Nentilla.

Schéma général d'installation des postes de mesures.

\section{III. - GALERIE D'AMENEE DE L'USINE DE NENTILLA}

La validité de la méthode de détermination des pertes de charge par circulation d'air dans la galerie de Nentilla était à priori moins assurée que pour les autres ouvrages étudiés jusqu'alors, en raison de l'impossibilité de réaliser les mêmes nombres de Reynolds pour les 2 écoulements, qui se placent tous deux dans la zone de transition précédant le régime turbulent rugueux.

La comparaison a été effectuée sur le troncon $\mathrm{DE}$, de $500 \mathrm{~m}$ de longueur, visible sur la figure 8 .

Les pertes de charge mesurées au cours des 2 séries d'essais sont reproduites dans la figure 9 où les échelles des pertes de charge et du carré des débits pour les 2 écoulements sont dans le mème rapport. Par suite, l'égalilé des pertes de charge devrait se traduile par la superposition des points de mesure, relativement mal réaliscé ainsi qu'on peut le voir.

En fait, les résultats des essais à l'air doivent être corrigés du fait de la présence d'une épaisseur d'eau relativement importante sur le radier de la galerie: il en résulte d'une part une réduction de la section mouillée, d'autre part une altération de la rugosité du radier. Au cours des essais avec circulation d'air nous avions calculé la correction due à la réduction de section à partir des relevés d'épaisseur de la lame d'eau sur le radier. Cette correction était de $3 \%$. Quant à linfluence de la différence de rugosité entre la paroi d'eau et le radier, nous l'avions évaluée à $3 \%$ d'après le relevé de la hauteur moyenne des rides de la surface de l'eau. Les valeurs des coefficients $\lambda$ relatives aux essais à l'air et reproduites dans le graphique $n^{\circ} 5$ tiennent compte de ces corrections. On pent voir que, malgré cela, elles sont supérieures à celles déduites des essais avec circulation d'eau.

L'écart moyen entre les coefficients dẻduits des 2 séries d'essais est de $9 \%$. Cet écart est significativement supérieur à celui que nous attendions à priori, compte tenu de la différence

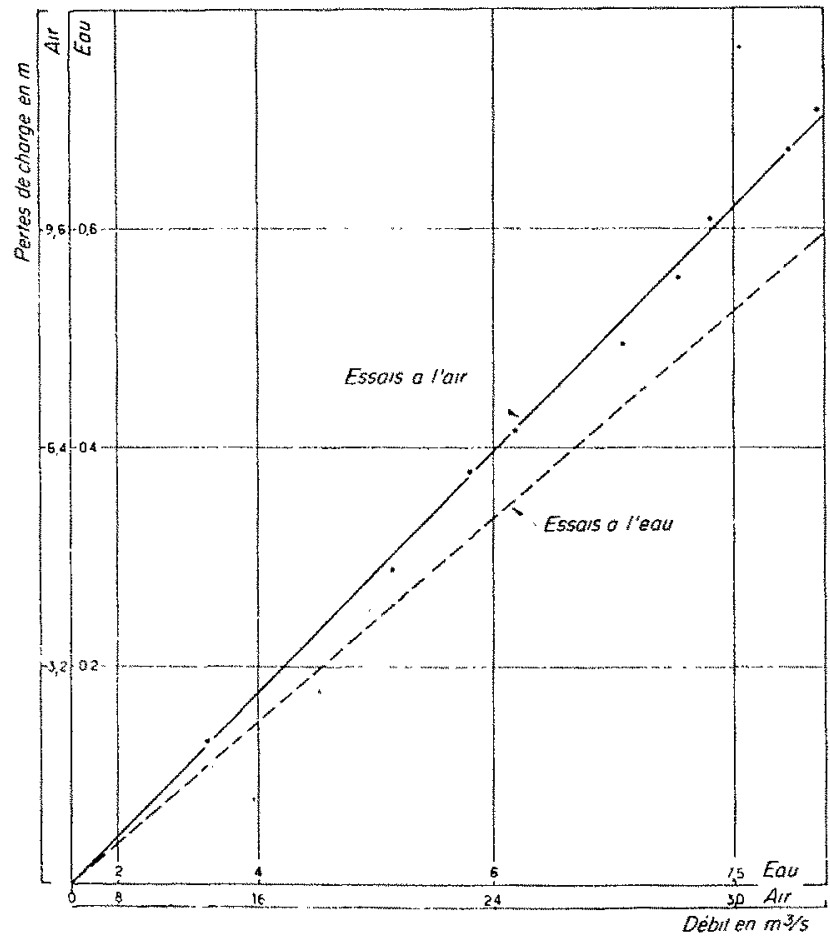

Fra. 9. -- Galerie de Nentilla. 
de turbulence. Nous basant sur les expériences de Colebroock, nous avions en efret évalué cet écart à 4 \% d'après la formule de Moody.

Nos estimations se trouvent ainsi trop pessimistes et en définitive les pertes de charge réelles se sont avérées inférieures de $5 \%$ à celles que nous avions estimées, compte tenu des essais de circulation d'air.
Cet écart se trouve à vrat dire compris dans la marge d'incertitude que nous évaluons à $\pm 5 \%$ dans les 2 séries d'essais. Toutefois, il pourrait peut-être se trouver justifié par le nettoyage des parois de la galerie dont la rugosité de certains troncons élait aggravée par des coulées de concrétions calcaires, sans doute disparues depuis la mise en eau.

\section{IV. - GALERIE DE PEYRAT-LE-CHATEAU}

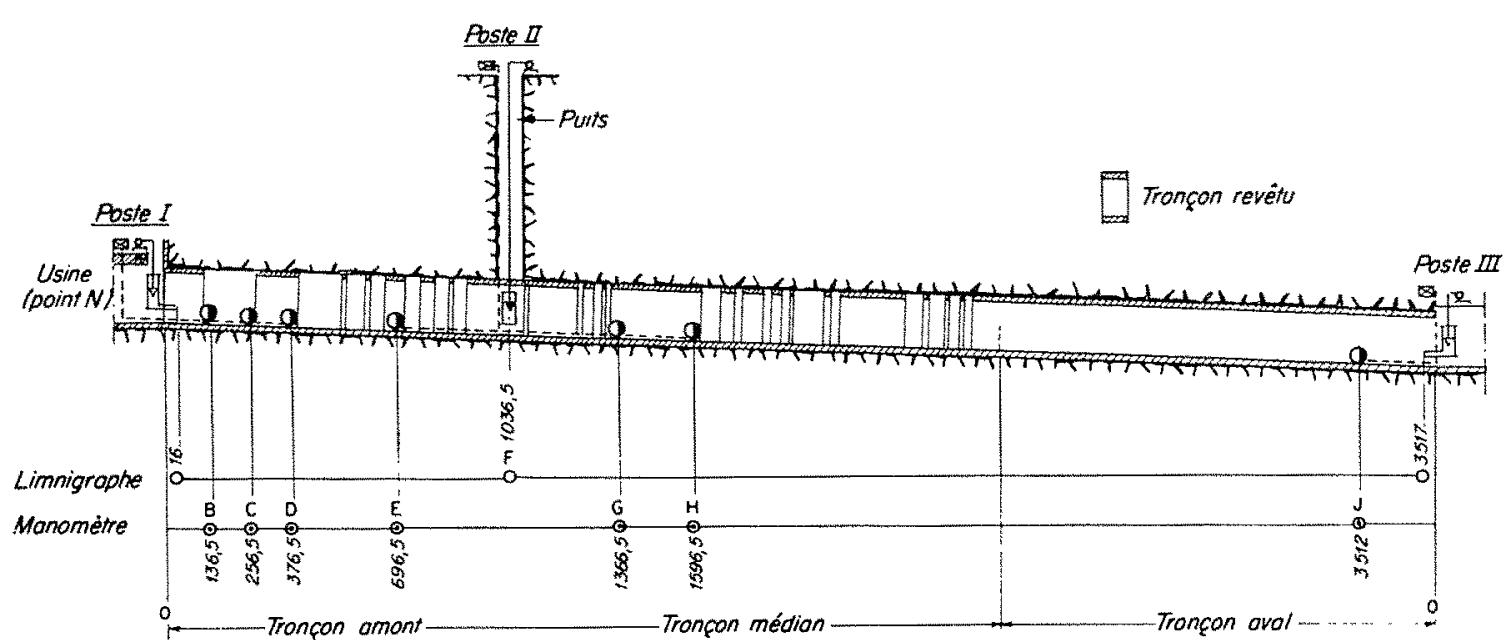

Frc. 10. - Galerie du canal de fuite de Peyrat-le-Château.

Schéma des installations pour les mesures des pertes de charge.

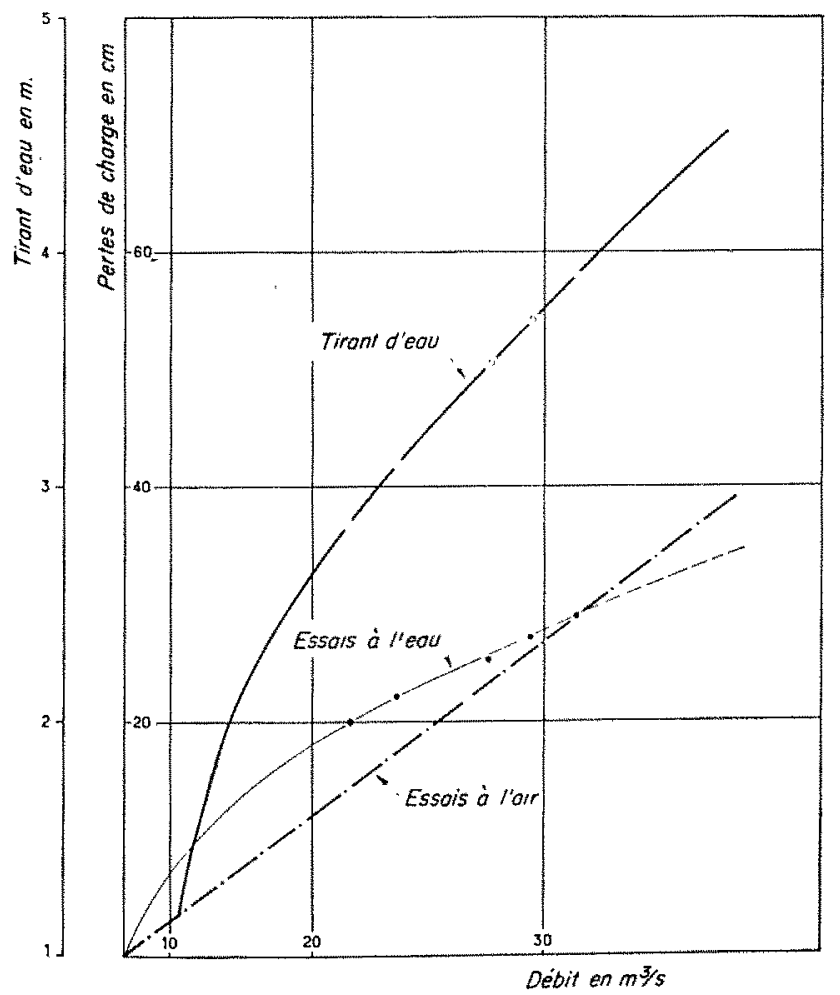

FIG. 11. - Galerie de Peyrat-le-Château.
L'utilisation de la circulation d'air pour la détermination des pertes de charge dans la galerie de Peyrat-le--Château était bien plus critiquable encore que pour la galerie de Nentilla. Si les parois étaient brutes et si, par suite, il ne se posait aucun problème de turbulence, la difficulté de transposer les résultats obtenus à l'air provenait du fait que la galeric de Peyrat-leChâteau fonclionne à écoulement libre.

Nous avons admis a priori que dans la samme des tiranls d'eau realisćs, les coeficients caractérisant la « rugosité » des parois de la galerie seraient égaux à ceux correspondant à l'écoulement en charge réalisé au cours des essais avec circulation d'air.

Les essais effectués depuis la mise on service de l'usine nous ont permis de déterminer la ligne d'eau correspondant à 5 valeurs du tirant d'eau comprises entre $2,80 \mathrm{~m}$ et $3,95 \mathrm{~m}$, soit entre $60 \%$ et $90 \%$ du tirant d'eau correspondant à la mise en charge. Le seul tronçon BC (fig. 10) comparable avec les essais précédents avait une longueur de $120 \mathrm{~m}$. Les pertes de charge mesurées y étaient relativement faibles; elles étaient en outre mesurées à partir de la différence d'indication de 2 manometres à transmission ćlec- 
trique, les dispositions locales ne se prètant pas à l'utilisation de manomètres différentiels. De ce fait, la précision de mesures est relativement médiocre; les pertes de charge ne sont guère connues à mieux de $\pm \mathbf{1 0} \%$ près pour chaque point.

La figure 11 reproduit les tirants d'eau moyens et les pertes de charge déduits des essais avec circulation d'eau. On peut y constater en particulier une dispersion de l'ordre de $\pm 5 \%$ des coefficients $\lambda$.
Ces valeurs sont comparées à celles résultant des essais avec circulation d'air dans le graphique de la figure 5 . On peut constater l'excellente concordance entre ces deux essais qui conduisent à des valeurs des coefficients $\lambda$ respectivement égales à :

$$
\begin{aligned}
& 0,745 \text { avec circulation d'air, } \\
& 0,765 \text { avec circulation d'eau, }
\end{aligned}
$$

soit environ $3 \%$ d'écart.

\section{V. - CONCLUSION}

En ce qui concerne les galeries non revêtues pour lesquelles on réalise relativement facilement la constance de la loi quadratique des débits pour la circulation d'air, les essais résumés cidessus permettent d'espérer unc prédispersion $\grave{a} \pm 5 \%$ en moyenne. Dans le cas des galeries lisses, la correction de Colebrook peut augmenter l'incertitude des résultats donnés par circulation d'air; pourtant, toutefois, les essais que nous avons effectués semblent donner une approximation du même ordre.

\section{DISCUSSION}

Président : M. REMENiERas

M. le Président remercie M. Bourguignon et rappelle que son exposé est la suite d'une communication antérieure au Comité Technique dont la première partie était consacrée à la méthode de prédétermination des pertes de charge dans une galerie parcourue par de l'eau ì partir de celles mesurées sur cette mème galerie parcourue par de l'air, et la deuxième, au compte rendu d'essais effectués suivant cette méthode (1).

A la demande des utilisateurs on a été amené à appliquer la méthode initialement prévue pour des galeries brutes de percement à des cas plus complexes : galeries revêtues et lisses, galeries à écoulement libre, etc.

D'autre part, les tronçons, souvent très courts, mis à la disposition des équipes d'essais, rendent les mesures difficiles, du fait de la faible différence manométrique à déterminer. Malgré ces difficultés, M. Bourguignos et ses dynamiques collaborateurs ont obtenu une précision surprenante et de très bons recoupements.

Cette étude a permis, chemin faisant, de mettre en lumière :

$1^{\circ}$ L'influence des poches d'air qui dans une galerie brute de mine, peuvent rester emprisonnées dans la calotte de la galerie et constituer une sorte de revêtement virtuel, plus lisse que la paroi réelle sur cette partie du profil;

20 Le peu d'intérêt économique d'un revêtement partiel des galeries, notamment du radier, tout au moins dans le cas de galeries de dimensions petites et moyennes.

(1) G. REMEnieras et P. Bourguignon: Quelques applications de la méthode de prédétermination des pertes de charge d'une canalisa. tion d'eau sous pression à partír de celles mesurées sur le même ouvrage parcouru par de l'air (résumé dans Mémoires et Travaux, vol. I, 1953, p. 22; article in extenso dans le Génie Civil du 15 thars 1953).
M. HaEgelen estime ce dernier résultat assez surprenant.

M. le Président en donne l'explication suivante : dans une petite galerie la diminution de la section mouillée, du fait du revêtement du radier d'une galeric brute, est importante en valeur relative; d'autre part, la turbulence dans l'ensemble de la section n'est qu'assez peu modifiée par ce revêtement partiel car les tourbillons qui se détachent de la fraction du profil demeurée brute se diffusent dans toute la section mouillée. Faut-il donc revêtir complètement toutes les galeries, demande M. HaEgelen?

En terrain de résistance mécanique et d'imperméabilité satisfaisantes c'est un problème d'optimum économique répond M. le Président.

M. HaEgelen pense que e'est peut-ètre pour des raisons d'usure que l'on revèt Ie radier; d'ailleurs, il ne tient pas pendant toute la durée de la galerie. A Bin-El-Ouidane, il a été nécessaire de refaire des radiers dans lesquels le béton n'avait pas tenu.

M. le Président indique que, par contre, dans certaines galeries d'amenée les vitesses sont telles que des radiers non revêtues peuvent se lisser par des dépôts à certains endroits et des érosions à d'autres.

M. Caquot suggère une synthèse des résultats des mesures de pertes de charge en section lisse et en section rugueuse, de manière à déterminer, par comparaison avec le calcul, les corrections que l'on devrait apporter aux diverses formules de pertes de charge.

M. Bounguigon pense qu'il est encore un peu prématuré de faire cette synthèse. En effet, d'après ces mesures, le coefficient de Strickler des galeries brutes varicrait entre 28 et 35 ce qui correspond à des écarts de 30 à $40 \%$ sur les pertes de charge. Celui des galeries revêtues en béton lisse exécuté, semble-t-il, suivant les mêmes procédés varie de 80 a 92 . Les tentatives effectuées pour 
relier la valeur du coefficient de Strickler de la galerie aux rugosités géométriques des parois n'ont pas donné jusqu'ici des résultats très homogènes.

D'autre part, les parois de certaines galeries se couvrent en service, de dépôts biologiques ou minéraux qui, à l'usine de Monceaux la Virole, ont entrainé le doublement de la perte de charge initiale dans un délai d'un an.

M. CaQvor précise que la confrontation qu'il suggère ne peut être profitable que si, parallèlement aux pertes de charge, on mesure la cause de celles-ci, et, en particulier, la rugosité proprement dite, défnie géométriquement, qu'il s'agisse de galeries brutes ou de galeries revêtues ou recouvertes de dépôts.

M. Charron indique d'après ses études de prix de revient des galeries soit brutes, soit bétonnées que, même en supposant que la galerie bétonnée conserve ses qualités au point de vue des pertes de charge, le revêtement n'est pas payant pour les petites galeries et mème pour les galeries moyennes. Il est peut-ôtre avantageux pour les grandes galeries comme celle de Randens et l'Isère-Arc, mais le problème est assez compliqué paree qu'il faut tenir compte du fait qu'il faut transformer les pertes de charge en pertes de $\mathrm{kw} / \mathrm{h}$, dont la valeur diffère suivant les époques. Le problème est donc très complexe.

Mais, et M. le Président confirme ce point de vue en le précisant, il $\mathrm{y}$ a des cas, assez nombreux, où le bétonnage s'impose par le fait de la mauvaise tenue ou de l'altérabilité à longue échéance du terrain; dans d'autres le projeteur estỉme devoir réduire au minimum le risque en prévoyant systématiquement le revêtement complet des galeries sans trop se soucier d'une opinion économique toujours un peu théorique.

M. Caguot observe que la discipline actuelle doit être d'implanter les galeries profondément dans le massif loin des vallées, ce qui augmente les chances de se trouver en terrain sain et d'éviter les revêtements en béton.

A ce propos M. Channon lait remarquer que l'on tend à faire le moins de fenètres possibles parce que l'on s'est rendu compte que le prix d'exécution d'une galerie au mètre linéaire n'etait absolument pas progressif en fonetion de la distance a la fenetre, mais qu'il ne devenait progressif qu'à partir d'une distance assez grande de celleci. A E. D. F. il y a très peu de galeries brutes. Dans les projets, elles l'étaient en grande partie, mais dans l'exécution, elles ne le sont plus que rarement.

M. Castelnau désirerait connaitre de combien le revêtement du radier dans une galerie déterminée modifie lo coefficient de rugosité.

M. Bounguignon indique que les essais comparatifs dans cet ordre d'idées n'ont été faits que dans les deux cas suivants :

1 Dans la galerie du Vénéon, où le radier nettoyé comportait des trous assez profonds, donc une importante rugosité naturelle, le revêtement virtnel constitué par une lame d'eau réduit de $12 \%$ les pertes de charge mesurées à l'air;

$2^{\circ}$ Dans le cas de Peyrat-Ie-Chatcau, où le radier constitué par le ballast de la voic de service n'avait pas été enlevé avant l'essai et était relativement peu rugueux, le revêtement en béton de ce radicr dans la zone occupée par le ballast n'a pratiquement pas changé la perte de charge : l'écart était certainement inférieur à $2 \%$.

M. LaBaye remarque que la réduction de $12 \%$ indiquée par M. Bourguignon englobe la réduction due à la rugosité absolue et eclle due à la diminution de section mouillée consécutive au revêtement, soil $3 \%$ d'après M. Bourguignon.

Nous recherchons :

Ingénieurs ayant quelques années de pratique dans l'aménagement de périmètres par irrigation et assainissement.

Ingénieurs pour participation à des études d'adduction d'eau et d'assainissement urbain.

Insónieurs débutants pour participation à des études d'Hydraulique agricole, d'adduction d'eau et d'assainissement urbain.

Ecrire: La Houille Blanche, $\mathrm{n}^{0} 101$. 